The authors express their appreciation of the help received from P. H. Hermans, Utrecht, The Netherlands. He ground material for us in his mill and supplied drawings of the mill. The authors are indebted to the machine shop of the Bureau for assistance in the design and construction of the mill at the Bureau, to Sanford Newman and Emil Borysko for the preparation of photomicrographs, and to Howard E. Swanson for the X-ray diffraction patterns.

\section{References}

[1] C. R. Burch, Proc. Phys. Soc. 59, 1, 47 (1947).

[2] R. Barer, A. R. H. Cole, and H. W. Thompson, Nature 163, 198 (1949).

[3] E. R. Blout, G. R. Bird, and D. S. Grey, J. Opt. Soc. Am. 40, 304 (1950).

[4] L. V. Foster, Anal. Chem. 21, 432 (1949).
[5] J. W. Rowen, C. M. Hunt, and E. K. Plyler, J. Research NBS 39, 133 (1947) RP1816.

[6] P. H. Hermans and A. Weidinger, J. Am. Chem. Soc. 68, 2547 (1946).

[7] S. Kiesskalt, Z. Ver. Deut. Ing. Beih. Verfahrenstechnik 1936, No. 1, 1 and DRP 619,662;620,721.

[8] D. Bachmann, Z. Ver. Deut. Ing. Beih. Verfahrenstechnik 1940, No. 2, 43.

[9] H. F. Launer and W. K. Wilson, Anal. Chem. 22, 455 (1950).

[10] K. Hess, H. Kiessig, and J. Gundermann, Z. Physik. Chem. B49, 64 (1941).

[11] K. Hess, E. Steurer, and H. Fromm, Kolloid Z. 98, 148, 290 (1942).

[12] K. Hess, Z. Ver. Deut. Ing. Beih. Verfahrenstechnik 1943, 61; Chem. Zentr. 1944, I, 6 and Chem. Abs. 39, $2905^{9}(1945)$.

[13] E. C. Yackel and W. O. Kenyon, J. Am. Chem. Soc. 64, 121 (1942).

[14] P. H. Hermans and A. Weidinger, Bul. Soc. Chim. Belg. 57, 123 (1948).

Washington, April 30, 1950.

\title{
Gravimetric Analysis of Exhaust Gas From Gas Turbine Combustion Chambers
}

\author{
By Fillmer W. Ruegg and Carl Halpern
}

\begin{abstract}
Because of the high air-fuel ratio used in combustion chambers of gas turbines, the concentration of products of combustion is so low that standard volumetric methods of analysis have proved unreliable. A gravimetric method of anaylsis was developed and applied to the problem, not to identify all of the constituents, but primarily to determine the efficiency of the combustion process. In this method the products of complete combustion, water and carbon dioxide, are separated from each other and from the remainder of the sample, which is then passed into a furnace to burn the combustible components. Carbon dioxide and water are determined by change of weight of solid absorbents. Experiments showed that the method may be used to provide accurate information about the magnitude of the loss of heat due to incomplete combustion, and partial information about the nature of the components of the exhaust gas.
\end{abstract}

\section{Introduction}

The primary objective of application of the combustion process in combustion chambers of gas turbines, and in fact in nearly all of its applications, is the release of energy in the fuel to heat the working fluid from its initial to its final temperature. Change of temperature of the working fluid is an indication of its enthalpy change, but temperature alone is inadequate to relate this enthalpy change to the energy available in the fuel. In order to obtain such a relation, calculation of a heat balance involving rates of flow as well as temperature must be made. It is inconvenient or perhaps impossible in some cases to make accurate measurements of these quantities, and it is in such instances that gas analysis may be called upon to supply the desired information about the efficiency of the combustion process. Even in cases where the heat balance can be calculated from experimental data, the measurements

\footnotetext{
1 The work described in this report was sponsored by the Bureau of Aero-
} nautics, Department of the Navy. are subject to uncertainties, and an accurate method of analysis of the exhaust gas is useful as an independent check. The analysis also provides information regarding the nature of the components of the exhaust, which may be useful in the design and development of combustion chambers.

Accurate analysis of exhaust gases from gas turbine combustion chambers is difficult because the fuel is burned with so much excess air that the concentration of the products of combustion is very low. Because of this dilution the more common methods of analysis, such as that employed in Orsat instruments, have proved unreliable. Gravimetric methods of analysis are known to afford precise results when dealing with small quantities, and it appeared that such methods could be applied to the problem of analysis of exhaust gas from combustion chambers for gas turbines. Jordan and Eckman [1] ${ }^{2}$ used gravimetric methods for the determination of gases in metals. The application to analysis of exhaust gas would depend on efficient separation of the prod-

\footnotetext{
${ }^{2}$ Figures in brackets indicate the literature references at the end of this paper.
} 
ucts of complete combustion, namely water and carbon dioxide, from each other and from the remainder of the sample. It was proposed to make the separation by using solid reagents to absorb water and carbon dioxide, and then to burn the combustibles remaining in the gas in a furnace over copper oxide. Water and carbon dioxide formed by combustion could then be separated and absorbed similarly. Use of a relatively large volume of sample would serve to minimize effects of errors in weighing on the accuracy of the determinations.

Separate determination of carbon monoxide, and relation of the amount so determined to the carbon dioxide and water produced by combustion in the furnace, wa: introduced to make the analysis more complete. Calculation of combustion efficiency from data of this type is possible, and the accuracy of the efficiency so calculated depends primarily on the completeness of separation of the various components and on the accuracy of the determination of the amounts of carbon dioxide and water before and after the treatment in the furnace. It was not proposed to identify and determine the quantity of each component of the exhaust gas, because it was considered that the time consumed and equipment required for such determinations would be prohibitive.

It is recognized that sampling technique is important if an accurate picture of the combustion process is to be had by gas analysis. As the primary interest was in the analytical technique, sampling was done to give an average sample rather than a picture of the combustion process at any particular point. The samples were taken at positions in the combustion systems where concentration gradients in the gas were at a minimum, the gas temperature was low enough that combustion reactions were probably stopped, and the problem of continued reaction in the water-cooled sampling probes did not arise.

The first application of the method at this laboratory was made in 1944, and since that time the investigation has been continued to determine the accuracy and the most efficient form of application of the method.

\section{Combustion Efficiency}

As one of the primary objects of exhaust-gas analysis is the determination of the efficiency of the combustion process, it is desirable to specify what is meant by combustion efficiency and what is required for its calculation. The primary interest in combustion problems is in the increase of enthalpy of the working fluid, and consequently combustion efficiency is usually expressed in terms of heat quantities, or in terms that are related to heat quantities. Numerous definitions have been proposed, and three that are commonly used are

$$
\eta_{b}=\frac{\Delta h_{\text {actual }}}{\Delta h_{\text {ideal }}}
$$

$$
\begin{aligned}
& \eta_{b}=\frac{\Delta T_{\text {actual }}}{\Delta T_{\text {ideal }}} \\
& \eta_{b}=\frac{f_{\text {ideal }}}{f_{\text {actual }}}
\end{aligned}
$$

The first definition relates the actual to the ideal change $o$ enthalpy, and this has been considered the orthodox definition. In practice, $\Delta h_{\text {actual }}$ is evaluated from a knowledge of the initial temperature of fuel and air and the temperature of the products of combustion, and from a knowledge of the specific heats of the components of the exhaust gas. Usually it is necessary to assume a composition, and the accuracy of the assumption will affect the accuracy of the evaluation of $\eta_{b}$. For many purposes, the assumption is made that the actual products have the same enthalpy-temperature relation as the products of complete combustion. The ideal change of enthalpy is generally replaced by the heat of complete combustion of the fuel, and therefore this definition is one that may be described as based on "stoichiometric" completeness. Heat of combustion of the fuel may be defined in several different ways, and generally the heating va'ue for combustion at constant pressure is used. There is no general agreement about the choice between the lower and upper heating value, and opinions differ whether these enthalpy changes should be evaluated with respect to some standard re.erence temperature, $T_{0}$, or to the initial temperature, $T_{1}$, of the reactants.

The second definition may be considered an approximation of the first. The third definition, which was proposed by A. L. London [2], relates the ideal amount of fuel required to produce the observed temperature after combustion to the actual amount of fuel burned. These definitions are used by some investigators in the field of combustion. Where the temperature of the products of combustion is high enough to cause dissociation of the carbon dioxide, water, oxygen and nitrogen, some investigators prefer to base the ideal rise of temperature or ideal fuel on the temperature that the gases attain when dissociation is considered and when equilibrium is established among the constituents of the exhaust gas. This is often referred to as "thermodynamically" complete combustion, and at temperatures over $1,550^{\circ} \mathrm{K}, \eta_{b}$ based on thermodynamic completeness will differ substantially from that based on stoichiometric completeness.

This brief discussion has indicated that there is no general agreement about the definition of combustion efficiency and that each can be calculated in various ways. Fortunately, it is unnecessary to further complicate the situation by injecting a different definition derived from data of chemical analysis. Equation 1 may be used as the basis for defining $\eta_{b}$ with suitable rearrangement to accommodate the type of data obtained from chemical analysis. Equations 2 and 3 require calculations that are lengthy and more complex. 
For the purpose of these calculations, it may be assumed that the air and fuel are brought together at temperatures $T_{a}$ and $T_{f}$, respectively, and that the weight ratio of fuel to air is $f$. Further, it may be assumed that the air and fuel mix at constant pressure with no change of phase and arrive at a common temperature $T_{1}$. A heat balance equation may be set up,

$$
f\left[h_{f}\right]_{T_{f}}^{T_{1}}=\left[h_{a}\right]_{T_{1}}^{T_{a}}
$$

and this may be solved for $T_{1}$. After the fuel and air mix, it is assumed that combustion takes place at constant pressure and that products of combustion of amount $(1+f)$ are formed.

Equation 1 may be written in the form

$$
\eta_{b}=\frac{\Delta h_{\text {actual }}}{\Delta h_{\text {ideal }}}=\frac{(1+f)\left[h_{b}\right]_{T_{1}}^{T_{2}}}{-f(\Delta H c)_{T_{1}}}
$$

which states that $\eta_{b}$ is the change of enthalpy of the products of combustion divided by the specific lower heat of combustion of the fuel for isothermal constant pressure reaction at the initial temperature. The negative sign indicates an exothermic reaction. It can be considered that the reaction takes place in two steps, the first of which is an isothermal reaction forming the actual products of combustion, and the second step is one in which the heat of the isothermal reaction is applied to the products to raise them to temperature $T_{2}$. Thus the numerator of eq 5 may be written

$$
(1+f)\left[h_{b}\right]_{T_{1}}^{T_{2}}=-\left(\Delta H_{\text {actual }}\right)_{T_{1}},
$$

where $\left(\Delta H_{\text {actual }}\right)_{T_{1}}$ is the heat of reaction at $T_{1}$, in which the actual products of amount $(1+f)$ are formed. In the real case, combustion is not complete, and the heat that can still be produced by continued reaction is determined by the difference between the heat of combustion of the fuel and the actual heat of reaction. Thus if the products of incomplete combustion were caused to react to completion at temperature $T_{1}$, the heat of this reaction would be determined by

$$
(\Delta H)_{T_{1}}=f(\Delta H c)_{T_{1}}-\left(\Delta H_{\text {actual }}\right)_{T_{1}}
$$

If eq 7 is solved for $\left(\Delta H_{\text {actual }}\right)_{T_{1}}$ and if its value is substituted in eq $5, \eta_{b}$ will be defined by

$$
\eta_{b}=1-\frac{(\Delta H)_{T_{1}}}{f(\Delta H c)_{T_{1}}},
$$

which is approaching a form amenable to application of data from exhaust gas analysis.

If all of the components of exhaust gas could be identified and determined quantitatively, it would be only necessary to find in the literature the values of the heat of reaction (or combustion) of the various components, and enter these values in the numerator, weighted according to the relative amounts of each. However, a complete determination of all the components would be extremely difficult, and some other means of arriving at a satisfactory answer is necessary. By standard thermochemical procedure we may write for oxidation of the combustible material

$$
\left.\begin{array}{rl}
-(\Delta H)_{T_{1}}= & -\left(\Sigma \Delta H f_{\text {products }}-\Sigma \Delta H f_{\text {combustibles }}\right)_{T_{0}} \\
& \left.+\left[h_{\text {combustibles }}\right]_{T_{0}}^{T_{1}}-\left[h_{\text {products }}\right]_{T_{0}}^{T_{1}}\right\}
\end{array}\right\}
$$

and for the fuel

$$
\left.\begin{array}{r}
-f(\Delta H c)_{T_{1}}=-\left(\Sigma \Delta H f_{\text {products }}-\Sigma \Delta H f_{\text {fuel }}\right)_{T_{0}} \\
+\left[h_{\text {fuel }+0_{2}}\right]_{T_{0}}^{T_{1}}-\left[h_{\text {products }}\right]_{T_{0}}^{T_{1}}
\end{array}\right\},
$$

where $\Delta H f$ is the heat of formation. The amounts of products and reactants are determined by the chemical equation for the complete oxidation of combustible and of fuel in amount $f$. Substitution of eq 9 and 10 in eq 8 leads to

$$
\eta_{b}=1-\frac{-\left(\Sigma \Delta H f_{\text {prod. }}-\Sigma \Delta H f_{\text {comb. }}\right)_{T_{0}}+\left[h_{\text {comb. }+0_{2}}\right]_{T_{0}}^{T_{1}}-\left[h_{\text {prod. }}\right]_{T_{0}}^{T_{1}}}{-\left(\Sigma \Delta H f_{\text {prod. }}-\Sigma \Delta H f_{\text {fuel }}\right)_{T_{0}}+\left[h_{\text {fuel }+0_{2}}\right]_{T_{0}}^{T_{1}}-\left[h_{\text {prod. }}\right]_{T_{0}}^{T_{1}}} .
$$

The magnitude of the change of heat of combustion with temperature for some of the more common fuels and products of combustion was calculated, and it was found that the change is small. The most extreme change, for hydrogen, is only about $3 \frac{1}{2}$ percent over the range from $0^{\circ}$ to $1,000^{\circ} \mathrm{K}$. Hence this effect may usually be neglected, and the enthalpy terms of eq 11 may thus be ignored.

Obviously, if the combustibles are not identified, it is not possible to use the value of their heats of formation, and hence it is necessary either to neglect this term in the numerator of eq 11, or to estimate its value. The heat of combustion of saturated hydrocarbons is generally about 90 to 97 percent of the sum of the heats of formation of their products of combustion. If for these hydrocarbons, the sum of the heats of formation of their products is used in eq 11 and if their heats of formation are ignored, an error will be introduced, amounting to about 0.03 percent to 0.1 percent for every 1 percent of combustion loss due to these compounds. In the case of olefins the error will be smaller, and for acetylene, the error thus introduced will amount to about 0.2 percent, but it is doubted that acetylene is present in exhaust gas in any significant amount. For partially oxidized compounds, such as carbon monoxide and formaldehyde, the error will be still larger, but these compounds may be identified and their quantity determined with relative ease, and the correct values can be used in the equation for $\eta_{b}$. 
From these considerations, eq 11 may be reduced to

$$
\eta_{b}=1-\left(\frac{\Sigma \Delta H f_{\text {products from combustibles }}}{\Sigma \Delta H f_{\text {products from fuel }}}\right) T_{0}
$$

The heats of formation of carbon dioxide and water are well established and are listed below:

$$
\begin{gathered}
\mathrm{C}+\mathrm{O}_{2} \rightarrow \mathrm{CO}_{2}, \Delta H_{298.10^{\circ} \mathrm{K}}=-2,137 \mathrm{cal} / \mathrm{g} \mathrm{CO}_{2}, \\
\mathrm{H}_{2}+\frac{1}{2} \mathrm{O}_{2} \rightarrow \mathrm{H}_{2} \mathrm{O}, \Delta H_{298.16^{\circ} \mathrm{K}}=-3,208 \mathrm{cal} / \mathrm{g} \mathrm{H}_{2} \mathrm{O} .
\end{gathered}
$$

All products and reactants are gaseous, except carbon, which is graphite. These heat values are listed on a weight basis, since the components are determined by weight in the analysis.

Let it be assumed that the weight of carbon dioxide originally in the exhaust gas sample, and the weight of carbon dioxide and water from combustion of unburned material have been determined. With this information, eq 12 may be written

$$
\eta_{b}=1-\frac{2137\left(\text { wt } \mathrm{CO}_{2}\right)+3208\left(\text { wt } \mathrm{H}_{2} \mathrm{O}\right)}{2137\left(\text { wt total } \mathrm{CO}_{2}\right)+3208\left(\text { wt total } \mathrm{H}_{2} \mathrm{O}\right)}
$$

where the terms in the numerator are from the unburned material, and the weight of total carbon dioxide is the sum of the carbon dioxide originally in the sample and the carbon dioxide from the unburned material. The quantity of total water is not determined experimentally but is calculated from the hydrogen and carbon content of the fuel. All terms of this equation may be divided by the heat of formation of carbon dioxide, which leads to

$$
\eta_{b}=1-\frac{\left(\text { w t } \mathrm{CO}_{2}\right)+1.50\left(\text { wt } \mathrm{H}_{2} \mathrm{O}\right)}{\left(\text { wt total } \mathrm{CO}_{2}\right)+1.50\left(\text { wt total } \mathrm{H}_{2} \mathrm{O}\right)} .
$$

Carbon monoxide is present in exhaust gas in relatively large quantity, and therefore it is necessary to correct for it. Let it be assumed that a separate analysis has determined the quantity of carbon dioxide from combustion of carbon monoxide. The ratio of the heat of combustion of carbon monoxide to the heat of formation of carbon dioxide is 0.719 , and hence eq 14 may be corrected to the following:

$$
\eta_{b}=1-\frac{0.719\left(\text { wt } \mathrm{CO}_{2} \text { from } \mathrm{CO}\right)+\left(\text { wt } \mathrm{CO}_{2}-\text { wt } \mathrm{CO}_{2} \text { from } \mathrm{CO}\right)+1.50\left(\text { w t } \mathrm{H}_{2} \mathrm{O}\right)}{\text { wt total } \mathrm{CO}_{2}+1.50\left(\text { wt total } \mathrm{H}_{2} \mathrm{O}\right)} .
$$

Similar modifications to eq 14 may be made for other compounds if the necessary information is determined about their quantity.

If the volume fractions of carbon dioxide and carbon monoxide in a gas sample are known in addition to the weight of free carbon dioxide, then the amount of carbon dioxide from combustion of the carbon monoxide may be calculated by the following equation:

$$
\text { wt } \mathrm{CO}_{2} \text { from } \mathrm{CO}=\frac{\text { vol } \mathrm{CO}}{\text { vol } \mathrm{CO}_{2}}\left(\text { wt } \mathrm{CO}_{2}\right) \text {. }
$$

The amount of total water may be calculated from the observed total weight of carbon dioxide by the equation,

$$
\begin{aligned}
\text { wt total } \mathrm{H}_{2} \mathrm{O}= & \left(\text { wt total } \mathrm{CO}_{2}\right) \times \frac{\text { at. wt } \mathrm{C}}{\text { mol. wt } \mathrm{CO}_{2}} \times \\
& \frac{\text { mol.wt } \mathrm{H}_{2} \mathrm{O}}{\text { mol. wt } \mathrm{H}_{2}} \times \frac{\text { wt H }}{\text { wt C }}
\end{aligned}
$$

where the weight percent hydrogen and carbon in the fuel is either estimated from its physical properties [3] or determined directly by methods such as outlined in reference [4].

Air-fuel ratio by gas analysis of this kind is based on the volume fraction of carbon dioxide in the dry gas as established by the volumetric analysis. When combustion is complete, carbon dioxide concentration may be used as an indication of the air-fuel ratio, and the relation

$$
\begin{aligned}
& (\mathrm{vol} \mathrm{CO})_{\eta_{b}=100}=\mathrm{vol} \mathrm{CO} \mathrm{CO}_{2} \times \\
& \frac{\text { wt free } \mathrm{CO}_{2}+\mathrm{CO}_{2} \text { from combustibles }}{\text { wt free } \mathrm{CO}_{2}}
\end{aligned}
$$

may be used to establish the concentration for the hypothetical case of complete combustion. This equation is not strictly correct, but its accuracy is sufficient for the present purpose. Air-fuel ratio may then be calculated by the equation

$$
\frac{1}{f}=\frac{240 \mathrm{C}}{\left(\operatorname{vol} \% \mathrm{CO}_{2}\right)_{\eta_{b}=100}}+7.15(1-\mathrm{C}),
$$

where $\mathrm{C}$ is the weight fraction of carbon in the hydrocarbon fuel. This equation may be derived from the chemical equation describing the relation among reactants and products of complete combustion of hydrocarbon fuels in air. If a gas sample representative of average conditions in the combustion chamber is desired, then the air-fuel ratio by gas analysis may be used as a criterion in judging the quality of the sample.

\section{Experimental Apparatus}

The combustion train used in this laboratory was patterned after that described by Wagman and Rossini [4].

A diagram of the assembled apparatus is shown in figure 1, in which $A$ represents a source of com- 
pressed air; $B$, control valve; $C, 3$-gal jar filled with activated alumina; $D$, mercury manometer; $E$, quartz combustion tube containing copper oxide wire; $F$, electric heater; $G$, female Pyrex connection, standard taper; $H$, graded seal, quartz to Pyrex; $I$, Pyrex absorption tube containing in order, anhydrone and ascarite; J, Pyrex absorption tube containing in order ascarite and anhydrone; $K$ Pyrex absorption tube containing phosphorus pentoxide. Components of the system $B$ through $K$ comprise the purification section. $L$ is a three-way stopcock; $M$, 16-liter stainless-steel sample container; $N$, three-way stopcock; $O$, Pyrex glass cold trap; $P$, female Pyrex connection, standard taper; $Q$ and $R$, electric heaters; $S$, quartz combustion tube packed with copper oxide wire; $T$, graded seal, quartz to Pyrex and $U$, flow meter; 1 , Turner absorption bottle containing in order drierite and phosphorus pentoxide, separated by asbestos; 2, Turner absorption bottle containing in order ascarite and phosphorus pentoxide, separated by asbestos; 3 and 5, Turner absorption bottles containing in order anhydrone and phosphorus pentoxide, separated by asbestos; 4 and 6 , Turner absorption bottles containing in order ascarite and phosphorus pentoxide, separated by asbestos. These bottles may be closed by turning the body under the cap. Temperature is measured by Chromel-Alumel thermocouples placed along the combustion tubes. Components $O$ through $U$ and absorption bottles 1 to 6 comprise the analysis section. The combustion tube is made of quartz; the length is $100 \mathrm{~cm}$; the internal diameter $20 \mathrm{~mm}$; and the wall thickness $5 \mathrm{~mm}$. Only the last $55 \mathrm{~cm}$ are heated in the furnace. The Turner absorption bottles that contain the reagents were modified by attaching $12 / 5$ male spherical joints to the caps. The cold trap was made of Pyrex glass, the inner tube of which was wrapped with glass wool, and a side arm was used to insert a copper-constantan thermocouple to measure the temperature of the gas. The freezing mixture, which gives a temperature of $-78^{\circ} \mathrm{C}$, was prepared by adding crushed dry ice to a mixture of equal weights of carbon tetrachloride and chloroform.

The balance used has a capacity of $200 \mathrm{~g}$ and a sensitivity of $0.1 \mathrm{mg}$. Before weighing the absorption bottles, the lubricant was carefully washed from the ball joints with carbon tetrachloride, and then the whole surface was washed with ethyl alcohol. The bottles were allowed to rest in the balance case for 20 min before weighing. A closed absorption bottle containing ascarite was used as a counterpoise.

The gas sampling tube consisted of an outer nominal 1/8-in. standard iron pipe with an inner 1/8-in. thin-walled copper tube. This sampling tube, or probe, was cooled by passing water through the space between the tube and pipe.

The stainless steel container had an internal volume of 1,000 in. $^{3}$ and was fitted with a needle valve at each end and with a compound vacuumpressure gage. Gas was drawn through the inner tube of the water-cooled probe and pumped into an evacuated tank by a diaphragm pump to a pressure

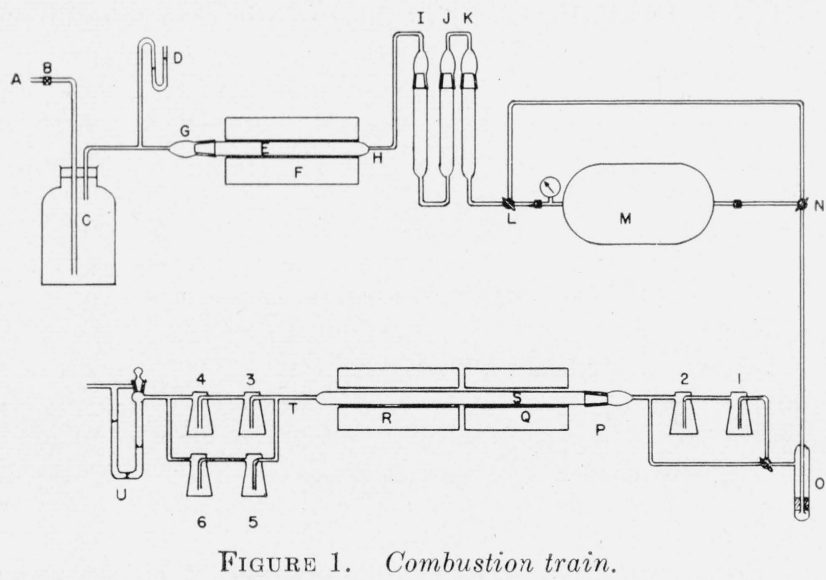

of about $30 \mathrm{lb} / \mathrm{in}^{2}$ gage. In some cases samples were collected at combustion-chamber pressure (usually near atmospheric) by connecting the evacuated container directly to the probe and opening the valve.

The volume fraction of carbon dioxide in the sample was found by analyzing a portion in an improved Orsat apparatus as described by Shepherd [5]. Colorimetric tubes described by Shepherd [6] were used to determine the carbon monoxide content. These tubes contain palladium sulfate and ammonium molybdate on silica gel. Passage of gas over this material produces a change of color, and a comparison with color standards indicates the concentration of carbon monoxide in the gas. The colorimetric tubes used in this investigation were designed and calibrated for passage of $90 \mathrm{ml}$ of gas at the rate of $1 \frac{1}{4} \mathrm{ml} / \mathrm{sec}$, and the change of color indicated concentration of carbon monoxide from 0.0025 to 0.020 percent by volume. Usually it was necessary to dilute a portion of the exhaust gas with air and pass $90 \mathrm{ml}$ of the mixture through the tubes. The concentration of carbon monoxide in the exhaust gas was then calculated from the observed concentration in the mixture.

\section{Analytical Procedure and Results}

Air was passed through the system until the furnaces reached the operating temperature of $1,000^{\circ} \mathrm{C}$, the absorption bottles were put in place, and then the exhaust gas was passed through the analyzing section of the system. When the gas sample in the container was at atmospheric pressure, it was displaced into the analysis section by air from the purification section. For samples at higher pressure, the gas was discharged into the analysis section, and the rate was controlled by adjustment of the needle valve on the sample container. The sample rate in most experiments was $400 \mathrm{ml} / \mathrm{min}$. After the sample was passed through the cold trap and bottles 1 to 4 , inclusive, the condensate in the trap was volatilized by gentle heating and flushed directly into the furnace and thence to bottles 5 and 6 by air from the purification section. All bottles were removed, and the combustion tubes were then 
allowed to cool while the flow of air was continued.

While this part of the analysis was in progress, a small sample of the exhaust gas was analyzed for its carbon dioxide and carbon monoxide content. These data, in conjunction with the observed increase of weight of absorption bottles $2,3,4$, and 6 were used to calculate combustion efficiency. Change of weight of bottle 5 was not measured since water vapor condensed in the trap and susequently was absorbed in bottle 5. Hence it was necessary to estimate the quantity of water from combustion of condensed hydrocarbons, and this was done on the assumption that the condensed material was original fuel. Equation 17 was used with the change of weight of bottle 6 to calculate the water of combustion in bottle 5 .

Experimental results from a series of tests in which 80 octane unleaded gasoline was burned are presented in table 1 , in which the observed changes of weight of the various absorption bottles are listed under the numbers that identify the bottles in figure 1 . Change of weight of absorption bottle 6 was corrected by subtracting $8 \mathrm{mg}$, as it was found that this amount of carbon dioxide was held up in the trap, some as gas and some occluded or dissolved in the condensate. This correction was established by flowing exhaust gas samples of the same size and composition (32 liters containing about $3 \mathrm{vol} \%$ carbon dioxide) through a cold trap at $-78^{\circ} \mathrm{C}$ and then warming and flushing the condensate into two absorption bottles by a stream of air free from carbon dioxide, water, and combustibles. The increase of weight of the ascarite bottle varied in four tests from 9.2 to $10.4 \mathrm{mg}$ and averaged $10 \mathrm{mg}$. It was estimated that approximately $6 \mathrm{mg}$ of this observed change was due to gaseous carbon dioxide remaining in the trap, and $2 \mathrm{mg}$ due to adsorbed hydrocarbons. Subtracting these estimated corrections from the observed change of weight of $10 \mathrm{mg}$ left $2 \mathrm{mg}$ of carbon dioxide dissolved or occuluded in the trap. The tests were made without flushing the cold trap with air before volatilizing the condensate, and the correction to bottle 6 in these tests was considered to be $8 \mathrm{mg}$.

TABLE 1. Analyses of exhaust gas

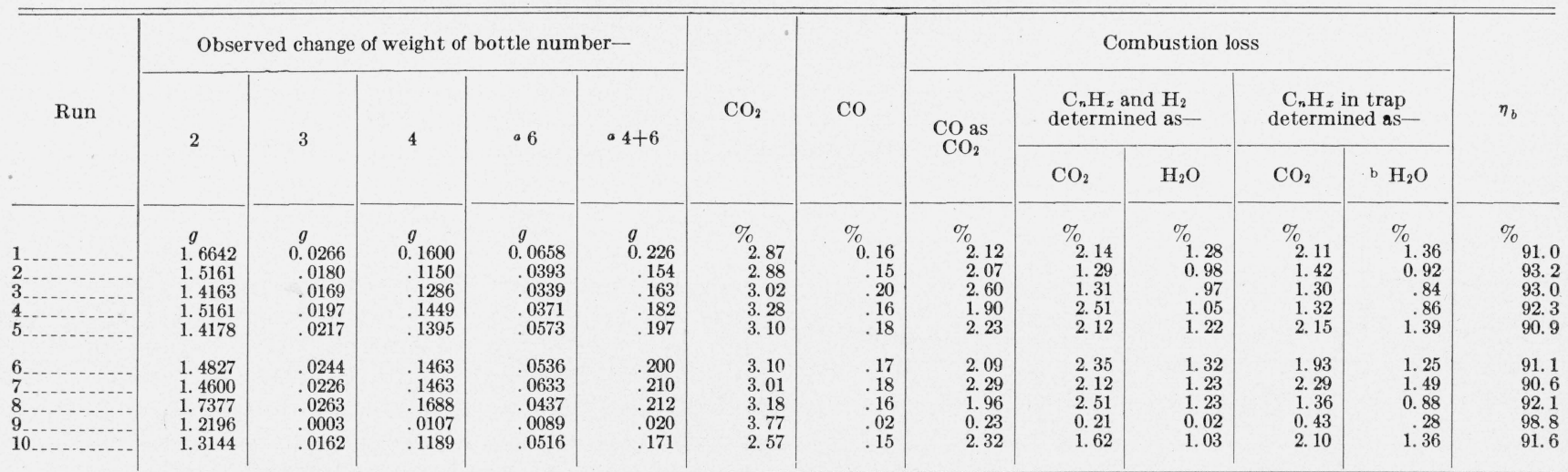

a Corrected for $8 \mathrm{mg}$ of $\mathrm{CO}_{2}$ retained in cold trap. weight.

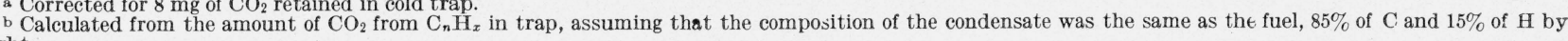

An independent check on the amount of carbon dioxide occluded in the trap was obtained by a volumetric method. As before, about 32 liters of exhaust gas, containing 2 percent of carbon dioxide by volume in this case, was passed through a trap at $-78^{\circ} \mathrm{C}$, and the trap was purged at low temperature with air free from carbon dioxide. It was then connected to an evacuated container (with a small trap at $0^{\circ} \mathrm{C}$ between) and warmed to about $+80^{\circ} \mathrm{C}$. The small trap at $0^{\circ} \mathrm{C}$ was used to collect water and some combustibles. It was realized that some carbon dioxide would possibly be held up in the small trap, but probably in insignificant amount. The gas collected in the evacuated container was analyzed by treating with alkali in an Orsat apparatus, and the contraction in volume was considered to be the volume of carbon dioxide from the cold trap. Five tests of this type were made, giving an average volume of $0.9 \mathrm{ml}$ and an average weight of $1.6 \mathrm{mg}$ of carbon dioxide. The weight of carbon dioxide was estimated from the observed volume. The average amount occluded from about 32 liters of gas containing 2 percent by volume of carbon dioxide was thus $1.6 \mathrm{mg}$. These results are in good agreement with the gravimetric determination of $2 \mathrm{mg}$, when the difference of carbon dioxide concentration in the samples is considered. If $2 \mathrm{mg}$ is considered applicable to the data of table 1 , neglect of this factor would have decreased the indicated $\eta_{b}$ by about 0.1 percent. This correction, however, may be dependent on the carbon-dioxide content of the exhaust gas, the apparatus used, rate of flow, etc., and probably must be determined for the special conditions under which the analysis is made.

Loss of combustion efficiency was calculated from the experimental data for the various types of combustible material, and the results are presented in table 1. These data indicate for these samples a combustion loss due to carbon monoxide of about 2 percent, that from hydrocarbons and hydrogen that passed through the trap about 3 percent, and that from combustibles of higher molecular weight retained in the trap also about 3 percent. 


\section{Accuracy of the Method}

Three samples of known composition were prepared and were diluted with air until the resulting mixtures contained components in concentrations that are considered typical of exhaust gases from combustion chambers. If it is assumed that these mixtures of known composition resulted from burning a fuel (gasoline) containing 85 percent of $\mathrm{C}$ and 15 percent of $\mathrm{H}$ by weight, then the combustion efficiencies would have been 94.7, 98.5, and 87.6 percent. The degree to which these values could be duplicated by the results of the analyses would constitute a check on the method.

The dependency of the values of combustion efficiency, which it is desired to check, upon the ratio of carbon to hydrogen in the fuel is slight. If the fuel that had been burned to give the first sample had been propane, which consists of 81.7 percent of $\mathrm{C}$ and 18.3 percent of $\mathrm{H}$, then the efficiency would have been 95.1 percent instead of the figure 94.7 percent for gasoline.

Data on the samples of known composition, and on the analyses of these samples, are given in table 2. The cold trap was not used in these tests. The agreement of the values of combustion efficiency calculated from the analytical results, with those calculated on the basis of the known composition, indicates that the method is capable of yielding reliable values of efficiency.

TABLE 2. Analyses of prepared samples

\begin{tabular}{|c|c|c|c|}
\hline \multicolumn{4}{|c|}{ Sample composition } \\
\hline $\begin{array}{l}\text { Sample number } \\
\text { Mole percent of } \mathrm{CH}_{4} \\
\text { Mole percent of } \mathrm{CO} \\
\text { Mole percent of } \mathrm{H}_{2} \\
\text { Mole percent of } \mathrm{C}_{2} \mathrm{H}_{4} \\
\text { Mole percent of } \mathrm{CO}_{2} \\
\text { Calculated comb. eff. } \\
\text { a }\end{array}$ & \begin{tabular}{c}
1 \\
$2.35 \pm 0.05$ \\
$2.60 \pm 0.05$ \\
$2.58 \pm 0.05$ \\
\hdashline 92.47 \\
$94.7 \pm 0.1$
\end{tabular} & $\begin{array}{c}2 \\
0.46 \pm 0.025 \\
.58 \pm 0.025 \\
51 \pm 0.025 \\
98.45 \\
98.5 \pm 0.1\end{array}$ & $\begin{array}{l}\quad 3 \\
\text { 4. } 15 \\
4.20 \\
4.20 \\
87.45 \\
87.6\end{array}$ \\
\hline
\end{tabular}

\begin{tabular}{|c|c|c|c|c|c|c|c|c|c|c|c|}
\hline \multirow[b]{2}{*}{ Run number. } & \multicolumn{11}{|c|}{ Analytical results } \\
\hline & 1 & 2 & 3 & 4 & 5 & 6 & 7 & 8 & 9 & 10 & 11 \\
\hline Sample number & 1 & 1 & 1 & 1 & 2 & 2 & 3 & 3 & 3 & 3 & 3 \\
\hline Sample rate, $\mathrm{ml} / \mathrm{min}$ & 400 & 400 & 400 & 400 & 400 & 400 & 150 & 250 & 600 & 800 & 800 \\
\hline Vol. $\mathrm{CO}_{2}$, after dilution, $\%$ & & & 2.7 & 2. 3 & & 2.4 & 2. 6 & 2. 3 & 2.5 & 2. 9 & 2.5 \\
\hline $1 / f$ & 30 & 110 & 74 & 87 & 84 & 86 & 70 & 80 & 73 & 65 & 73 \\
\hline Vol. CO (exptl.), \% & & & 0.090 & 0.059 & & 0.013 & 0.04 & 0.04 & 0.04 & 0.05 & .04 \\
\hline Vol. CO (known), \%b & & & .076 & .064 & & .011 & .13 & .11 & .12 & .14 & .12 \\
\hline $\mathrm{CO}_{2}$ bottle $2, \mathrm{~g}$ & 2.0491 & 0.4785 & .5304 & .4647 & 0.4970 & .3831 & .2788 & .2843 & .3939 & .4588 & .3782 \\
\hline $\mathrm{CO}_{2}$ bottle $4, \mathrm{~g}$ & 0.1082 & .0242 & .0288 & .0239 & .0065 & .0058 & .0416 & 0380 & .0572 & .0625 & .0548 \\
\hline $\mathrm{H}_{2} \mathrm{O}$ bottle $3, \mathrm{~g}$ & .0643 & 0127 & .0174 & .0152 & .0036 & .0037 & 0181 & .0211 & .0237 & .0289 & .0278 \\
\hline Comb. eff. from analytical datac & 94.7 & 95.2 & 94.6 & 94.6 & 98.6 & 98.3 & 87.7 & 87.6 & 88.2 & 88.4 & 87.2 \\
\hline Comb. eff., calc. ${ }^{\circ}$ & $94.6 \pm 0.1$ & $94.6 \pm 0.1$ & $94.6 \pm 0.1$ & $94.6 \pm 0.1$ & $98.6 \pm 0.1$ & $98.6 \pm 0.1$ & 88.4 & 88.4 & 88.4 & 88.4 & 88.4 \\
\hline
\end{tabular}

$\triangle \eta_{b}$ calculated, using eq 11 . Fuel assumed to have been $85 \%$ of $\mathrm{C}, 15 \%$ of $\mathrm{H}$, and $\Delta H c / \Sigma \Delta H f=0.965$

b In runs 7 to 11 , the known value of $\mathrm{CO}$ was used in the calculation of $\eta_{b}$.

o $\eta_{b}$ calculated, using eq 15 .

Tests on the third sample of known composition were made to determine the effect of rate of flow on the analytical results. At the rate of $800 \mathrm{ml} / \mathrm{min}$, the computed combustion efficiency was less consistent, and it was deemed advisable to stay below this rate for better precision. It was decided to establish a rate of $400 \mathrm{ml} / \mathrm{min}$ as a standard, since tests at this rate on the first two known samples were satisfactory. Determination of carbon monoxide was inaccurate in the tests of the third sample, probably because of the relatively high concentration of unsaturated material in the gas. Shepherd states [6] that unsaturated organic compounds interfere in the determination of carbon monoxide by this method. Combustion efficiency was computed using the known concentration in order to better determine the accuracy of the gravimetric part of the analysis.

Accuracy of the results of the tests of the prepared samples also may be determined by a comparison of the experimental with the known values of the ratio of the weight of carbon dioxide from combustibles to the free carbon dioxide in the sample. A comparison can also be made of values of the ratio of water from combustibles to free carbon dioxide. Combustion efficiency is determined by the values of these ratios, and an accurate determination is desirable.

Average values of these ratios are plotted in figure 2 , and it may be seen that the value of the carbon dioxide ratio determined by analysis in the combustion train is very close to that calculated on the basis of the known composition. Values of the ratio of water from the combustible material to the weight of carbon dioxide in the third sample is somewhat higher than the known value, but agreement is good for the first and second samples.

Another check on the accuracy of the analytical method was by a comparison of combustion efficiency by gas analysis with that established by heat balance across several combustion chambers. Analyses were made using the original method in which the cold trap was not used, and it was on the basis of these tests that the procedure was revised.

Seventeen analyses were made on exhaust gas from combustion of gasoline, and seven were made on gas from combustion of kerosine, or fuel similar to kerosine. When gasoline was used, combustion 


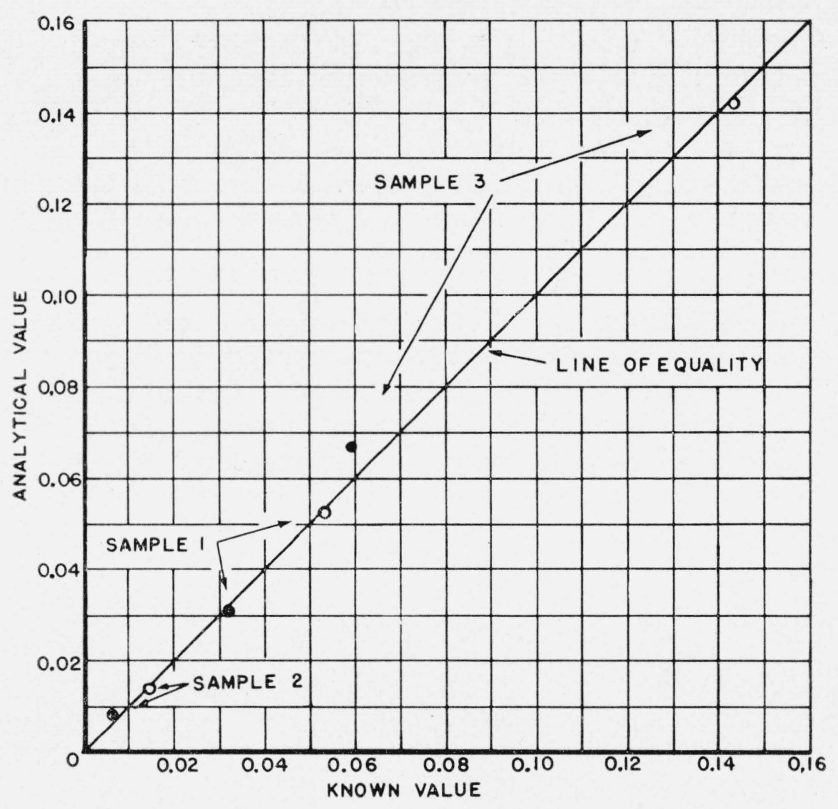

Figure 2. Results of analyses of prepared samples.

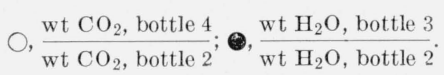

efficiency indicated by gas analysis was on the average 2 percent higher, and after correcting the heat balance observations for such factors as loss of heat from the system and thermocouple, incomplete recovery of dynamic temperature, and the small water content of the air, the difference was reduced to 1 percent. When kerosine was burned, the difference was considerably greater and in the same direction. In this case it was considered that the major part of the difference was caused by loss of combustible material from these samples by condensation in the sampling probe, vessel, and connecting tubes. This explanation did not seem to apply to the samples from combustion of gasoline. In this case it was found that combustibles of higher molecular weight were adsorbed in bottles 1 and 2 . It was for this reason that the procedure was revised to include the use of the cold trap. By this means these combustibles could be separated from the gas before passage through the adsorption bottles and later passed directly into the furnace.

A series of tests was made in which identical samples were analyzed with and without the cold trap in the train, and $\eta_{b}$ was on the average about 1 percent lower when the trap was used. Since the previous comparison of $\eta_{b}$ by heat balance and by gas analysis had indicated that gas analysis was high by about 1 percent, results of these tests indicated that agreement would be excellent with the improved method of analysis.

A comparison of the data from the two methods of analysis indicated that approximately 45 percent of the combustibles of higher molecular weight were adsorbed in bottles 1 and 2 when the trap was not used. It was found that recovery was possible by flushing the absorption reagents with air, but it appeared that recovery was only 55 percent complete after $3 \mathrm{hr}$ of flushing. The correction of $2 \mathrm{mg}$ for adsorbed hydrocarbons that was mentioned in the preceding section in connection with the amount of carbon dioxide occluded in the trap was also estimated from these data.

It is known that formaldehyde is present in exhaust gas under some conditions of combustion. However, only a trace of formaldehyde was found in one of a number of samples taken from various combustion chambers operating on different fuels. If the cold trap is used in the combustion train, presence of formaldehyde will not introduce a large error, as it will condense in the trap and be burned with other condensed compounds. A small error will result, since the heat of formation of formaldehyde is neglected when $\eta_{b}$ is calculated. If the cold trap is not used, experiment has shown that formaldehyde is completely absorbed by the ascarite in absorption bottle 2 , and a separate analysis would be necessary.

Blank runs. Fourteen blank runs were made to determine the experimental error to be expected in these analyses. Air, freed from carbon dioxide, water, and combustibles in the purification section of the train, was passed for a period of $3 \mathrm{hr}$ at the rate of $400 \mathrm{ml} / \mathrm{min}$ through the train. The cold trap was not used. Results from these runs showed that the average error was of the order of $1.5 \mathrm{mg}$. Some determinations also were made of the reproducibility of weighing. A clean absorption bottle packed with reagents was first weighed, and then handled as usual, including greasing, followed by the cleaning operation. Air was not passed through the bottle. Change of weight was no more than $0.2 \mathrm{mg}$ in the weighing experiments. It is probable that passage of the large quantity of air through the bottles was responsible for the larger experimental error determined above.

\section{Conclusion}

A gravimetric method of analysis was developed and found applicable to exhaust gas from gas-turbine combustion chambers. Combustion efficiency determined by this method agreed with that calculated from the composition of prepared samples to about $1 / 2$ percent; combustion efficiency obtained by the analysis of actual gas samples agreed with that calculated by heat balance to better than $1 / 2$ percent. It is concluded that the gravimetric method may be used to provide accurate information about the magnitude of the loss of heat due to incomplete combustion, and partial information about the nature of the components of the exhaust gas.

\section{References}

[1] Louis Jordan and J. R. Eckman, The determination of oxygen and hydrogen in metals by fusion in vacuum, BS Sci. Pap. 18, 445 (1925) S514.

[2] A. L. London, Gas-turbine plant combustion-chamber efficiency, Trans. ASME 70,317 (1948). 
[3] Thermal properties of petroleum products, NBS Misc. Pub. M97 (1929).

[4] D. D. Wagman and F. D. Rossini, A note on the macroanalysis of carbon and hydrogen by combustion, J. Research NBS 32, 95 (1944) RP1577.
[5] Martin Shepherd, An improved apparatus and method for the analysis of gas mixtures by combustion and absorption, BS J. Research 6, 121 (1931) RP266.

[6] Martin Shepherd, Rapid determination of small amounts of carbon monoxide, Anal. Chem. 19, 77 (1947).

Washington, February 13, 1950.

\title{
Absorption of Near-Infrared Energy by Certain Glasses
}

\author{
By Jack M. Florence, Charles C. Allshouse, Francis W. Glaze, and Clarence H. Hahner
}

\begin{abstract}
An analysis has been made of the absorption bands of various glasses by calculation of the internal transmittances and surface losses. The wavelengths, in microns, associated with broad absorption bands have been measured, and the active vibrating groups causing the absorption are as follows: $2.7, \mathrm{CO}_{2} ; 2.75, \mathrm{OH} ; 2.75, \mathrm{CO}_{2} ; 2.9, \mathrm{OH}$ associated $; 3.5, \mathrm{CO}_{3}^{--}$; 3.65, $\left(\mathrm{NO}_{3}{ }^{-}\right) ; 3.8, \mathrm{Si}-\mathrm{O}$ bond; 4.0, $\mathrm{CO}_{3}^{--} ; 4.15,\left(\mathrm{NO}_{3}^{-}\right) ; 4.25, \mathrm{CO}_{2} ; 4.45, \mathrm{Si}-\mathrm{O}$ bond; and 4.7, OH associated. The greatest loss of infrared energy for a number of glasses is caused by surface losses, rather than by true internal absorption.
\end{abstract}

\section{Introduction}

\section{Gaseous Components of Glasses}

Glasses contain, in solution and as bubbles, small amounts of gaseous components. These are residues of the decomposition products of the original batch materials or the result of contact with the furnace atmosphere. The gaseous components in the body of the glass may be considered as present in two conditions: namely, in true solution and as a part of the internal structure. The internal transmittances for any glass, when plotted against wavelength, will contain absorption bands in the region of the spectrum in which these gases absorb. The intensity of the absorption bands depends upon the concentration and the absorbing power of the gas.

Because the surfaces of many glasses are chemically active toward the atmosphere, they also become of importance in the transmission of nearinfrared energy. Weyl $[1]^{2}$ explains that the configuration of the ions in the surface layer of a glass is not identical with that in the internal structure. He has explained the presence of $\mathrm{OH}^{-}$in a glass as being a surface phenomenon. The surface of silica glass has a film of $\mathrm{OH}^{-}$formed from the hydrolysis of Si-O-X bonds, where $\mathrm{X}$ is either an alkali or an alkaline earth ion. The $\mathrm{X}$ forms a water soluble salt that is leached out and replaced by a hydroxonium ion $\left(\mathrm{H}_{3} \mathrm{O}^{+}\right)$. The surface contains those $\mathrm{Si}-\mathrm{O}-\mathrm{Si}$ bonds that were originally present in the glass and a number of the $\mathrm{Si}-\mathrm{OH}$ groups.

The above explanation may be carried further by $\mathrm{X}$ forming first a hydroxide and then a carbonate. Taking a lithium silicate glass as an example, it follows:

\footnotetext{
1 This project was supported by the Department of the Navy, Bureau of Ships.
}

Figures in brackets indicate the literature references at the end of this paper

$$
\begin{aligned}
-\mathrm{Si}-\mathrm{O}-\mathrm{Li}+2 \mathrm{HOH} & \longrightarrow \mathrm{SiO}-\mathrm{OH}_{3}+\mathrm{LiOH} \\
-\mathrm{SiO}-\mathrm{OH}_{3} & \longrightarrow \mathrm{SiOH}+\mathrm{HOH} \\
2 \mathrm{LiOH}+\mathrm{CO}_{2} & \longrightarrow \mathrm{Li}_{2} \mathrm{CO}_{3}+\mathrm{HOH}
\end{aligned}
$$

The products of the reaction, plus any sorbed water from the atmosphere, remain on or near the surface of the glass.

Roman, Marboe, and Weyl also found that glass surfaces containing $\mathrm{Ba}^{++}$and $\mathrm{Pb}^{++}$ions, among others, showed decreasing hygroscopicity with decreasing ionic potential [2]. It has been found that glasses containing $\mathrm{BaO}$ and $\mathrm{PbO}$ have a high transmissivity for infrared energy for wavelengths of 2.5 to $4.5 \mu[3,4]$.

\section{Characteristic Wavelengths of the Vibrating Groups}

The identification of the vibrating groups has been made from the knowledge of the gaseous components that may be expected to be in a glass and their characteristic wavelengths. It has been possible to identify the vibrating groups to be expected in a glass through a knowledge of the decomposition products of the batch materials used and their characteristic absorption bands. The precise location of the wavelengths at which these absorption bands occur has been amply discussed by Herzberg [5].

The wavelength of the fundamental vibration of $\mathrm{OH}$ in water vapor has been identified at $2.66 \mu$. The hydroxyl vibration becomes broader and stronger and moves to a longer wavelength when "hydrogen bonding" occurs [5]. The substitution of a silicon atom for one of the hydrogen atoms of the $\mathrm{HOH}$ dipole also creates a difference in the internuclear distance of the $\mathrm{OH}$ bond, thereby changing the observed wavelength for this vibration. The wavelength for the $\mathrm{OH}$ vibration in the vapor phase, obtained from glasses, is $2.75 \mu$. When the water is 